\title{
STATE OF ARTS OF MONUMENTAL STONES DIAGNOSIS AND MONITORING
}

\author{
Piero Tiano, and Cristiano Riminesi
}

\begin{abstract}
National Reserach Council Institute for the Conservation and Valorisation of Cultural Heritage, Via Madonna del Piano 10, Building C, 50019 Sesto Fiorentino, Firenze, Italy-p.tiano@icvbc.cnr.it; cristiano.riminesi@cnr.it
\end{abstract}

\section{Commission II}

KEY WORDS: Portable diagnostic tools, Imaging techniques, Spectroscopy techniques, Moisture content, Salt content, , Biodeterioration, Hardness, Scheduled maintenance.

\begin{abstract}
:
The conservation and maintenance of a monumental stone building is a complex aim where different disciplines are involved. First step is concerning the determination of the state of conservation of stone material present, than determine its modification on time, as such and after conservation treatments applied on: cleaning, protecting, strengthening. In order to fulfill such objectives suitable parameters must be selected and the most appropriate diagnostic techniques for their quantitative evaluation operated. In this context, the determination of the surface water absorption, moisture content, colour variation and mechanical properties are important parameters for the control, and the monitoring over time, of the state of conservation of the monumental stone surfaces. These parameters are strongly related not only to the stone characteristics but also to the evaluation of products's performance: efficiency and durability. Their rate of variation, determined in monitoring campaigns, is fundamental for elaborate by properly predictive model a schedule maintenance protocol.
\end{abstract}

\section{INTRODUCTION}

The experience of the practice in conservation intervention, applying methods on monumental stones, inform us that the same product provides different performances when applied on different substrates and different environmental context.

The state of conservation of the object (monumental stone, or other works of art) and its conservation problems must be clear and well characterized. For this purpose, an exhaustive diagnostics campaign should be performed together with a monitoring session in order to define the real conditions and the potential risk for its conservation. Indeed, it is not yet possible to indicate a product with ideal features and, in order to evaluate the efficacy and the harmfulness of a treatment, it is necessary to face every specific case on the basis of a preliminary diagnostic study and a preventive laboratory control.

In case of application of conservation treatments, these must be tested for evaluate their performance: efficiency and durability directly on site. A pilot monumental substrate in which this choice finds the final response is the right answer. In this preliminary step, conservation treatments are tested in small representative areas: cleaning, desalination, application of consolidants and/or water-repellent treatments. In this approach it is possible to outline some advantages and drawbacks; the former include that the products are tested in the real case using the real state of conservation of the substrate in its environmental exposition (Mecchi et al., 2008). The latter involve the difficulty to locate a surface with enough homogeneity in order to apply the different products with the same conservation level and exposure.

\section{NON-DESTRUCITIVE TECHNIQUES}

Non-destructive testing (NDT) covers a wide group of analysis' techniques used to evaluate the properties of a material, part, product, or system without materially affecting the integrity of the object under test. Consequently it is a highly valuable technique that can save both money and time in product evaluation, troubleshooting, and research. This approach is particularly suitable for the diagnosis of Cultural Heritage assets, due to their uniqueness. Most commons NDT methods used in this field include: ultrasonic pulse velocity, remote visual inspection, eddy-current testing (for metal artworks), multispectral imaging (VIS-UV-IR), infrared reflectography and laser scattering methods.

In this paper we illustrate some NTD portable methodologies employed by the ICVBC (ICVBCmobile Laboratory) for the diagnosis and monitoring of monumental cultural heritage and for evaluate the performance of conservation treatments.

\subsection{Imaging techniques}

\subsubsection{Multispectral photographic techniques}

The multispectral photographic techniques called imaging are non-invasive and portable methods of analysis of works of art (paintings, canvas, wall paintings and also painted stone artifacts). The imaging techniques present in the ICVBCmobile Laboratory are the following: Ultraviolet reflected photography (UVr); Ultraviolet fluorescence photography (UV); Near Infrared photography (IR); Visible Induced Luminescence photography (VIL); Infrared False Color (IRFC); Ultraviolet False Color (UVFC)

UVr can be used for characterization of pictorial material. As visible photography, ultraviolet radiation is sent on the surface of the object which can absorb or reflect the radiation, according to material and substances on it. The images are acquired by means of a digital camera (Canon EOS 400D). The object is irradiated homogenously and for this purpose a set of two flashes are used (Flash Quantum mod. T5D). In order to detect only UV radiation reflected from the target, pass band filters are inserted ahead of the two flashes (Schneider Optics $\mathrm{B}+\mathrm{W} 403 \mathrm{UV})$. The images obtained are elaborated by means of computer graphic program in a black and white photo. The classical UV imaging technique is used for spatial identification of varnish and protective treatment, but also binders and repaintings. Organics materials, on every surface such as mural paintings, canvas, marble and wood, can be detected in such way non-invasively. Fluorescence is the phenomena of the 
emission of light from a substance that has absorbed energy through an electromagnetic radiation. The UV fluorescence occurs when the absorbed radiation is in the UV region of the spectrum, and thus invisible to the human eye, and the emitted light is in the VIS region. This fluorescence is detected from the camera equipped with filters that cut UV and IR reflected from both the target and the environment. Furthermore, this emission can increase with ageing, due to intensification of chemical interactions, and this property could be exploited to distinguish latest repainting.

The IR imaging technique is used for characterization of pictorial material, identification of pigments and in some cases to study f preparatory drawings. The IR radiation is directed on the target which can absorb or reflect the radiation, according to material and substances on it. The spectral range is between 730 $\mathrm{nm}$ and $1000 \mathrm{~nm}$ (camera sensor limit). Diffusion of light, as scattering, decrease with the use of long wavelengths. This phenomenon makes pictorial layers transparent to the radiation. This technique examines in depth the painted layers (until to tens of microns) given information on the presence of preparatory drawing.

In the Visible Induced Luminescence photography, as in the UV fluorescence photography, the VIS radiation is directed on the target and for some pigments an emission in a NIR range occurs. The emission from flashes is filtered to obtain only visible radiation (Schneider Optics B+W 486 UV/IR digital blocking filter) and the images are acquired by means of a digital camera with the inner low-pass filter removed. The target must be irradiate homogenously and as example the emission happens around $910 \mathrm{~nm}$ for Egyptian Blue in mixture containing such pure pigment.

With the introduction of digital photography the IFRC and UVFC techniques have become a post processed techniques. They could be used for identification of pigments and materials. In IFRC the IR is superimposed on VIS image. In visible image RGB channels are shifted, the blue channel is replaced with green and green channel with red. The red channel is replaced with IR image. Instead, in the UVFC technique UV is superimposed on visible image, and the green channel is replaced with blue and red channel with green (Figure 1).

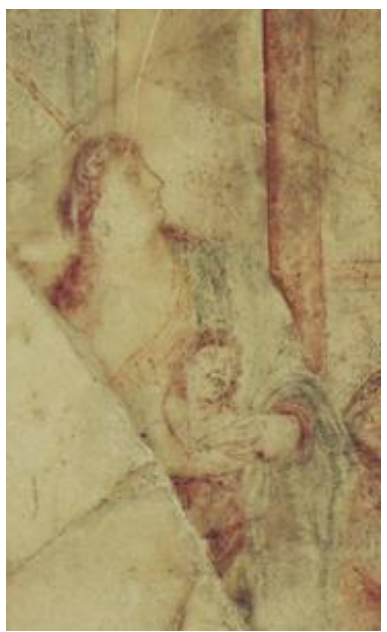

(a)

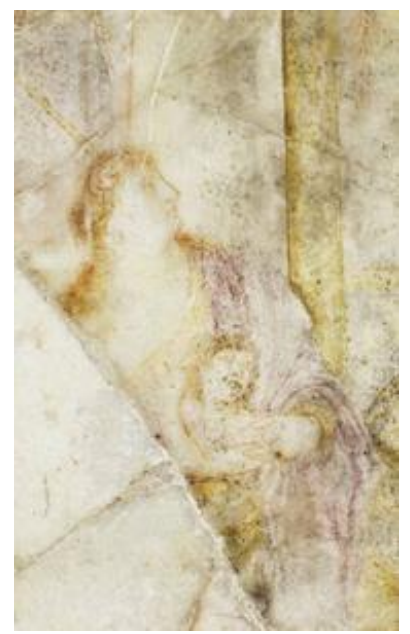

(b)
Figure 1. Example of Ultraviolet False Color (UVFC) imaging technique. (a) VIS image and (b) UVFC image

The blue channel is replaced with ultraviolet image. In this manner a false colours image is obtained. In this manner a false colours image is obtained. In this way pigments, having different chemical composition but looking very similar in visible region, can be differentiate by their different aspect in IR and UV range (i.e. the IRFC technique lets to differentiate between lapis and azurite).

\subsubsection{IR Thermography}

The IR thermography is a detection technique that allows capturing images, called thermograms, in the infrared thermal range of the electromagnetic spectrum. These images are got by means of a proper detector capable of measuring the emissivity of a substrate, or the radiation of the heat emanating from the body as a function of its temperature. Nowadays, this detector is installed in a commercial thermocamera quite similar to a normal digital photocamera for size and weight. All structures, with a temperature above absolute zero, emit radiations in the infrared range and the camera is able to measure their temperature by measuring the intensity of the emitted radiation (based on the Stefan-Boltzmann law).

Different materials emit heat unevenly following different temperatures reached; this is registered by the camera that provides a false colour image. In these imagines it is possible distinguish the different materials and possible decay phenomena (water infiltration, rising dampens, detachment, etc.) (Figure 2).

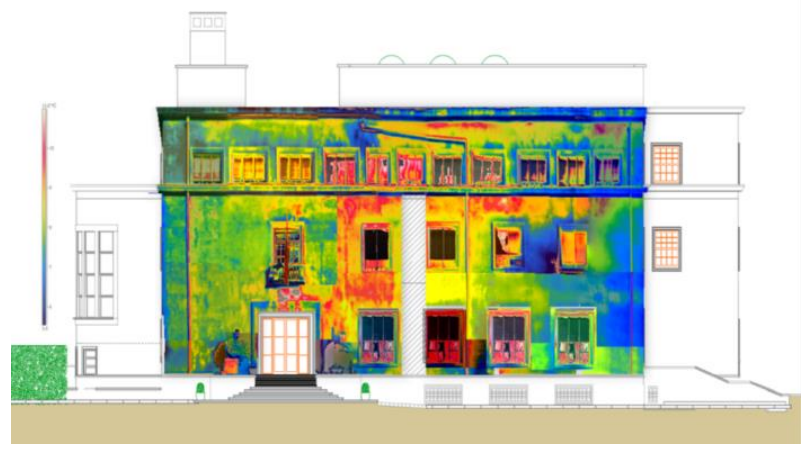

Figure 2. IR thermography image representing the water infiltration (in blue) form the right side of the roof (Brigate Palace, Tirana, Albania)

The thermographical analysis does not require physical contact between the measuring instrument and the object investigated, and this allows non-invasive and non-destructive controls. This technique has become essential in the diagnosis preliminary to the restoration of buildings or monuments and for monitoring the efficacy and durability of treatment or intervention. In fact, it:

- detect the texture of the walls below the layer of plaster. This allows to evaluate the morphology of the wall elements and then derive their stratigraphy, enhancing pre-existing forms suppressed and incorporated into the masonry, raising possible structural changes, constructive anomalies or hidden cavities; - detect structural deterioration, such as cracking state of the structure, presence of humidity, due to water infiltration or rising dampness, the latter could be source of biodeteroration processes

- detect, in conservation interventions, the humidity of extractive poultice, used for the reduction of the salinity content from walls, before its removal;

- detect the path of the injected formulates or mortar under pressure, when performing consolidation interventions.

\subsubsection{Chlorophyll fluorometers imaging}

Chlorophyll fluorometers are highly sensitive research instruments which give quantitative information on the quantum yield of photosynthetic energy conversion. Their measuring method is based on the Pulse Amplitude Modulation (PAM) technique. The essentials features of the PAM fluorometry are the extremely selective detection system to distinguish between 
the fluorescence excited by the measuring light and the much stronger signals caused by ambient and actinic light. The imaging portable instrument (PSI Photon Systems Instruments) is specialized for the study of two dimensional heterogeneities of the photosynthetic activity.

PAM fluorometer provides not only images of chlorophyll fluorescence, but also images of all relevant chlorophyll fluorescence parameters using the Saturation Pulse method.

The Chlorophyll Fluorescence imaging in Pulsed Amplitude Modulation (CF-PAM) has been usefully used for assessing vitality changes of photosynthetic biodeteriogens on stone artifacts. The effectiveness of the in situ application of microwave heating treatment (MEM) for the impairment of the biological patinas was evaluated directly on site by controlling the photoautotrophic vitality trough the portable fluorometer (Riminesi C. et al, 2016; Mascalchi M., et al., 2015). In this way images of photosynthetic activity and its spatio-temporal variations can be detected (Osticioli I., et al., 2013).

The experimentation has been performed on different biological patinas of stone monuments (Marble statues in the cemetery of the Certosa's cloister of Firenze, Figure 3). The result achieved defines an optimized application protocol for the use of this imaging technique for in situ phototrophic patinas characterizations in conservation studies. Figure 4 illustrates the results of such acquisition before and after the microwave heating treatment. For the Chlorophyll the minimum fluorescence, $F_{0}$, and the maximum fluorescence yield $F_{m}$ are determined and the maximum quantum yield of PSII calculated $\left[\left(\mathrm{F}_{\mathrm{m}}-\mathrm{F}_{0}\right) / \mathrm{F}_{\mathrm{m}}\right]$

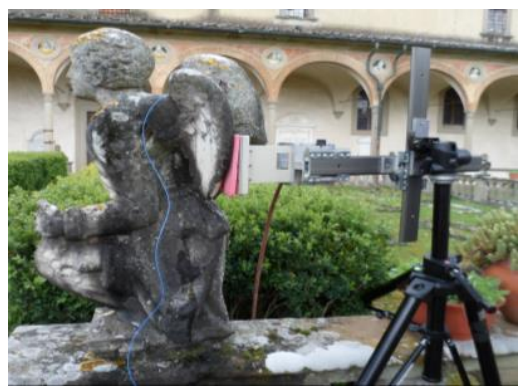

Figure 3. Microwave heating treatment on the biological patina growing on a marble statue
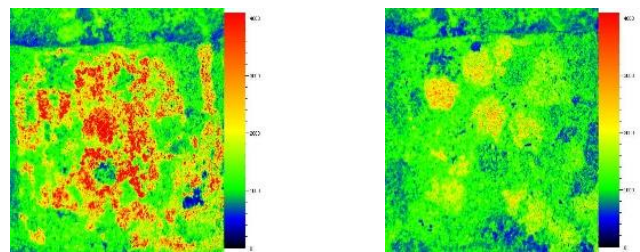

Figure 4. Maps of the maximum quantum yield of PSII calculated by $\left(\mathrm{F}_{\mathrm{m}}-\mathrm{F}_{0}\right) / \mathrm{F}_{\mathrm{m}}$, before (left) and after (right) the treatment.

\subsubsection{Ultra-Close Range Photogrammetry}

The Ultra-Close Range Photogrammetry (UCRP) system is contactless and totally non-invasive, and it can be used to record the surface pattern and for monitoring the effectiveness of cleaning treatment, as well as, the durability of protectives and consolidants (Barbetti I., et al., 2013; Manganelli Del Fà R., et al., 2014).

The UCRP is based on the same principles of classic photogrammetry, but is applied to a different scale: this technique allows to generate a RGB points cloud of a surface acquiring only three images (or more if necessary), defined triplet, shooting from different angles, of the same area. The size of the acquired area ranges from 2 to $20 \mathrm{~cm}^{2}$, based on the distance between the shots and the lens of the camera. The system is composed of a digital camera Canon EOS 7D equipped with calibrated Canon EFS $60 \mathrm{~mm}$ macro lens, which runs on a motorized bar $260 \mathrm{~mm}$ long (Figure 5), and of three dedicated software that allows to choose the right acquisition parameters, generate the point cloud, and elaborate the 3D model quoted in its $\mathrm{x}, \mathrm{y}$ and $\mathrm{z}$ components. The measurement accuracy on the $\mathrm{z}$ axis ranges between $30-50 \mu \mathrm{m}$ based on the light exposure and the reflectivity of the surface.

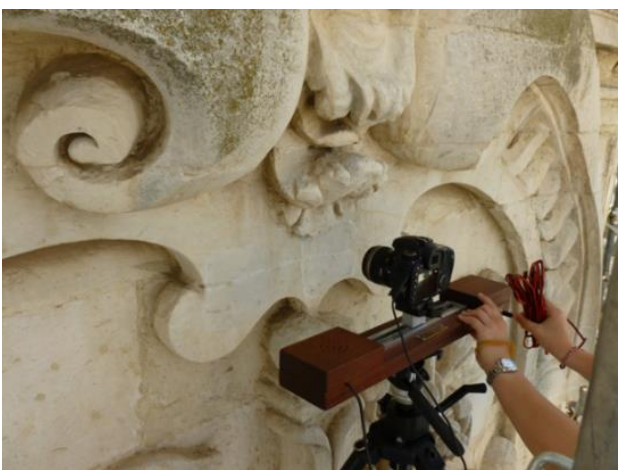

Figure 5. Measurement set-up of UCRP on the façade of the Lecce's Cathedral

By using commercial devices, it is possible to choose different focal lengths, e.g. the use of a $28 \mathrm{~mm}$ lens allows the reconstruction of larger areas after the acquisition of multiple shots, and the single models are mosaicked in order to obtain a single one.

The acquired images are processed by a dedicated software for generate a single points cloud through a specific algorithm; the points cloud generation process follows: image rectification, in order to eliminate geometrical and optical distortion; selection with a polyline of the area of interest; definition of the step resolution value; generation of a points cloud.

On the 3D model can be applied a texture (derived from the photographic image) that allows to appreciate colours changes. Once the acquisition and 3D model reconstruction have been done, the software, generates the Digital Elevation Model (DEM) choosing a suitable reference plane (UCS - Users Coordinate System) determined by means of the selection of the seed points on surface. DEM is the digital representation of the surface pattern respect to the selected UCS.

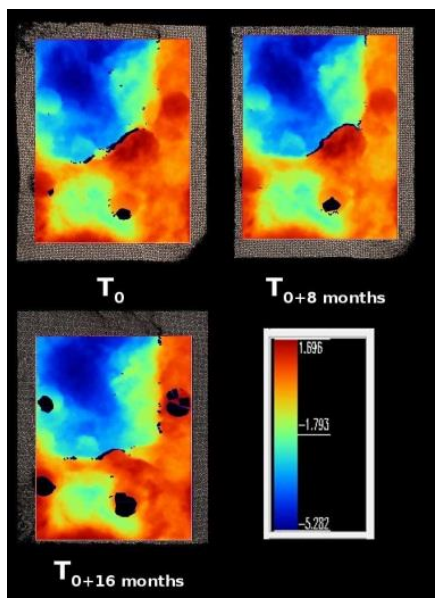

Figure 6. Differences in the elevation quotes (DEM) from images taken on the test surface of the calcareous stone (Santa Croce cathedral, Lecce) in time. 
By overlapping the same area acquired at different time, e.g. before and after a cleaning treatment, it is possible to compare the changes in pattern surfaces (Figure 6). The 3D profile of the quotes of the surface is extracted through the DEM of each models. The analysis of the frequency distribution of the quotes on the investigated area allows the extraction of some dimensional information on the surface pattern such as roughness or quote of particular areas (maximum and minimum values of elevation).

Choosing, by a polyline, a direction on the surface, it's possible to generate roughness profiles, whose coordinates can be exported in text format and elaborated with any software that allow their graphics processing.

\subsubsection{Evanescent Field Dielectrometry}

The Evanescent Field Dielectrometry (EFD) is a diagnostic methods based on electromagnetic measurement of the permittivity of the material under investigation. This method has been usefully employed for determining by non-invasive way the moisture and salts content inside materials (Olmi R., et al., 2006). The theoretical concept is based on the measurement of the dielectric contrast between water (about 80) and the host material in the microwave frequency range. The dielectric constant of typical materials used in masonry (brick, plaster, mortar and stone) is about 3-5. Thanks to the high dielectric contrast, it is possible to perform a powerful diagnostic of both the moisture content and the soluble salts inside the material up to $2 \mathrm{~cm}$ in depth. The measure is carried out in real time, needing some seconds to be completed.

The dielectric constant (real part of dielectric permittivity) determines the ability of the material to polarize itself, or to form and to orient electric dipoles when an external electric field is applied. This behaviour is quantified by the real part of permittivity; on the contrary the imaginary part is related to the dissipative effects due to friction acting between the electric dipoles while chasing the variations of the electric field. The imaginary part also depends on the electrical conductivity of the material, or rather from ions mobility. Therefore, a dry material containing salts can be characterized by a low electrical conductivity, even if the salts are present in high quantity.

The proposed system (SUSI ${ }^{\odot}$ tool, USA Patent, Bini et al., 2009) measures independently both the real part and the imaginary part of permittivity in the microwave frequency range. The support is investigated by the electric field that spills out from the aperture of the opened resonant cavity. In this case, the sensor is a truncated coaxial line closed on one side on the resonant circuit and on the other side opened on the material under investigation. Errore. L'origine riferimento non è stata trovata. shows the setup of the measurement system. It consists of a microwave signal meter (on the left in the picture), the probe (on the bottom in the picture), and a notebook on which is installed the software to control the instrument, as well as for the real time elaboration.

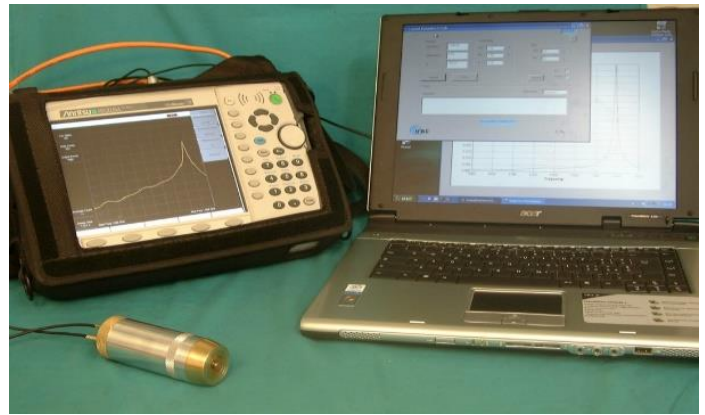

Figure 7. SUSI ${ }^{\oplus}$ tool

The measurement is performed keeping in contact the probe with the surface of the material under investigation (avoiding areas with risk of detachment or with others criticality). The measurement is averaged on a hemisphere $2 \mathrm{~cm}$ depth of radius into the material. Maps in terms of moisture content (MC) and salinity index (SI) can be elaborated from the data collected during the survey of the surface under investigation.
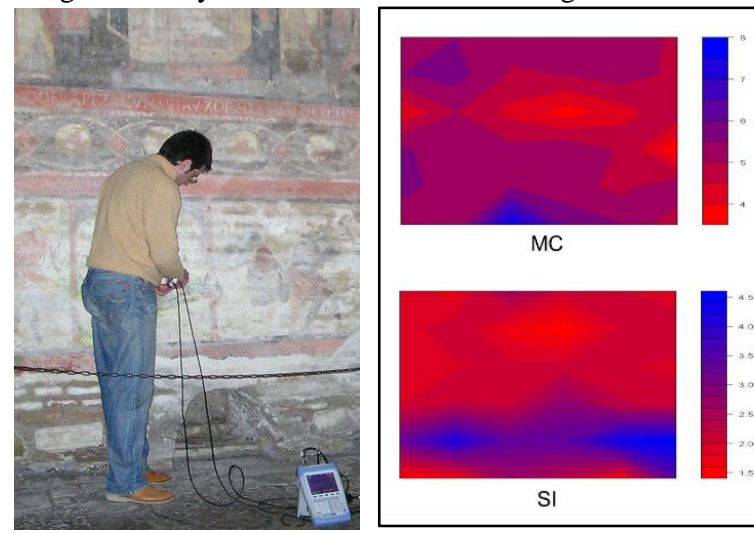

MC

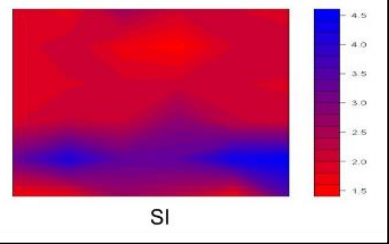

Figure 8. Maps of MC and SI on the wall paintings "The legend of St. Alex", S. Clement church, Rome

\subsection{Others techniques}

\subsubsection{Portable FT-IR spectroscopy}

The FTIR analyses can be performed on site by the use of the ALPHA-R spectrometer (Bruker Optik) (footprint $22 \mathrm{~cm} \times 33$ $\mathrm{cm}$, weight about $7 \mathrm{~kg}$ ). The interferometer inside the ALPHA$\mathrm{R}$ spectrometer (RockSolidTM) has a cubic corner mirrors; therefore, the sensitivity to vibrations is much lower compared with that of standard plane optics used in conventional Michelson's interferometers. To collect infrared spectra without touching the surface of the sample, a reflectance head with specular optics and long working distance (about $2 \mathrm{~cm}$ ) can be used. The integrated high-resolution camera allowed us to observe the measured spot and to record an image of it. The detector works at room temperature. The spot investigated was circular with a diameter of about $6 \mathrm{~mm}$. The spectral range covered by the instrument is $350-7,500 \mathrm{~cm}^{-1}$, with $4 \mathrm{~cm}^{-1}$ spectral resolution. The device has been usefully used to verify the presence and type of protective and consolidant treatments on one experimental wall placed into the archaeological area of Pompeii (Figure 9). 


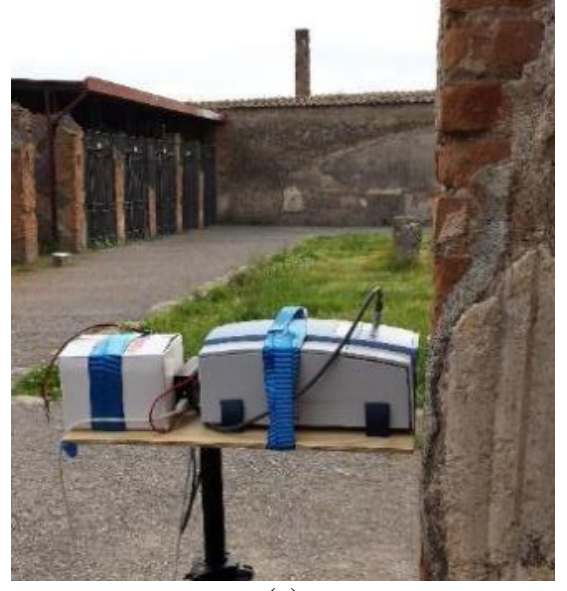

(a)
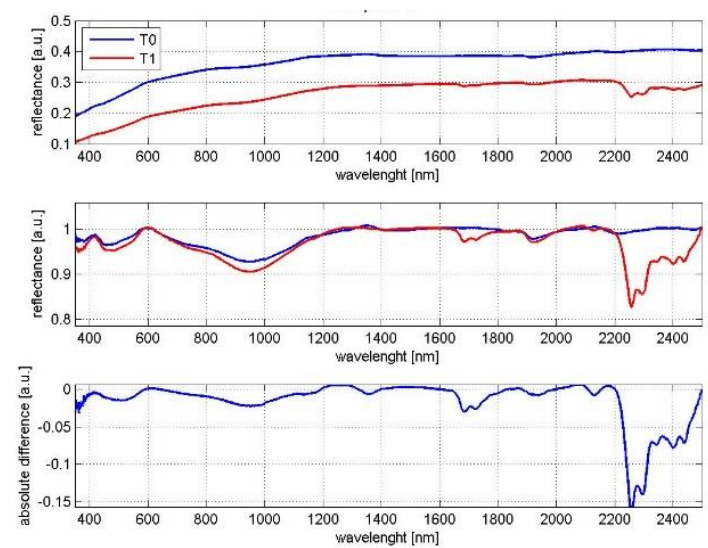

(b)

Figure 9. (a) Portable FTIR applied on the wall of the Macellum in the archaeological site of Pompeii; (b) Reflectance spectra on Campanian Ignimbrite before and after the treatment.

\subsubsection{Contact sponge method}

The contact sponge method is a water absorption test useful in evaluating differences induced by water repellents treatments (Vandevoorde et al., 2009; Tiano \& Pardini, 2004). The method consists in applying a soaked sponge with a controlled pressure on the surface to be tested. The water needed to soak the sponge is measured in advance; the soaked sponge is weighed, applied and kept in contact for 1-2 minutes (depends on the material porosity); then it is weighed again. The weight difference is a measure of the water absorbed by capillarity. The measures are carried out before $\left(\mathrm{T}_{0}\right)$ and after $\left(\mathrm{T}_{1}\right)$ the application of the water repellent treatments.

This very friendly portable system has been used for monitoring the behaviour of two different protectives applied on testing areas of the Ratto delle Sabine, a marble statue of Giambologna placed in the Loggia dei Lanzi (Piazza Signoria, Firenze, Italy), Figure 11. After the application of two repellent products the water absorption is decreased, even if not at the same level for each protective; monitoring the efficiency and the durability of the treatments we can see (Figure 11) that, after about 60 months, while product $S$ retains almost its water repellency the product $\mathrm{W}$ has loosed it for about $30 \%$.
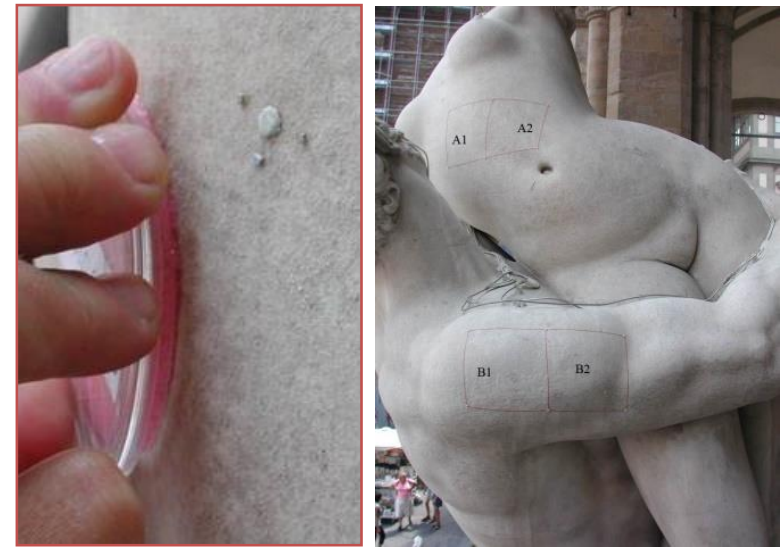

Figure 10. Contact sponge applied on a testing areas for the evaluation of water repellency of the protective treatments applied on the Marble statue of Ratto delle Sabine, Loggia dei Lanzi, Firenze, Italy

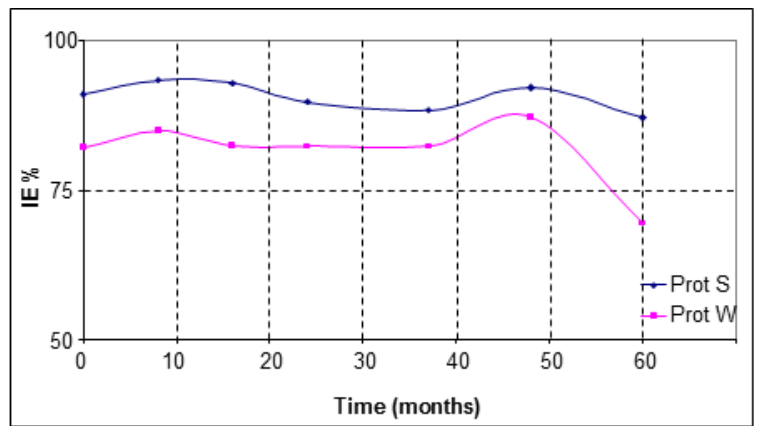

Figure 11. Water repellency monitoring, by the Contact Sponge, of the two protective treatments applied on the marble statue of Ratto delle Sabine

\subsubsection{Drilling Resistance Measurements System}

The Drilling Resistance Measurements System (DRMS) is a portable micro destructive method but is surely a technique that allows an effective on site assessment of the "hardness /cohesion" of natural or artificial stone, it lets to obtain real time cohesion profiles (drilling resistance versus depth) (Rescic S., et al., 2010). The system measures the force $(\mathrm{N})$ needed for drilling a micro hole $(3 / 5 \mathrm{~mm})$ and the penetration depth $(\max 5$ $\mathrm{cm})$ of the bit during drilling. The rotational speed and penetration rate are maintained constant and the operational software allows real-time display and recording of the cohesion profile in depth. The drilling resistance depends on mineralogical and structural characteristics of stone materials and it can be correlated with the traditional mechanical parameters (Exadaktylos et al., 2000; Fratini et al., 2006). The system has an application limit in presence of stone materials with abrasive constituents (such as granites) that quickly wear out the bit, producing a fictitious increase of drilling resistance. Due to this problem data corrections following specific step were pro-posed (Singer et al., 2000; Delgado Rodrigues \& Costa, 2004). Moreover, in presence of very hard (some kind of marbles) or very soft (calcareous tuffs) materials the drilling resistance values can exceeds the load cell range $(>100 \mathrm{~N}$ or $<1 \mathrm{~N})$. But due to the possibility to modify the two velocities we can anyway record a cohesion profile even in such materials. When the system is used to verify the possible consolidation action due to inorganic products, such as ammonium oxalate or barium hydroxide, the data recorded are not meaningful because these products, very similar to the stone composition, do not induce an evident increase in the drilling resistance. 
The advantages of the system are: it is the unique portable device that allows reliable direct measurements of the cohesion profile of stone materials, especially for onsite tests, so it is useful in the evaluation of consolidation treatments; the instrument and the data management are user-friendly, the speed of execution of one test (1-2 minute) and the versatility of the system allows to carry out measures in almost all worksite conditions. Furthermore, last but not least, the system could be useful for the preliminary identification of the test area where apply the conservation treatments. The drilling resistance measures allow to assess the heterogeneity of the material at depth (due to the presence of grains of different hardness) and on the outer surface (in case of natural or artificial crusts); moreover it quantifies the surface cohesion letting to identify either those areas which effectively need the conservative treatment and their penetration depth; therefore a optimization of the application method and of the product concentration is attained. The study case of the wall of the Etruscan tombs in the Sovana archaeological area (Grosseto, Italy) is reported as example; the tombs are carved in a red volcanic tuff with black scoria. This kind of stone materials, being a porous material with a high water absorption coefficient, is particularly subject to decay (mainly loss of material and powdering) due to environmental factors (temperature variations, water infiltrations, humidity, diffusion of soluble salts with efflorescence). For these reasons it needs consolidant and protective conservative treatments. Three areas were chosen for the drilling resistance tests (Figure 12).

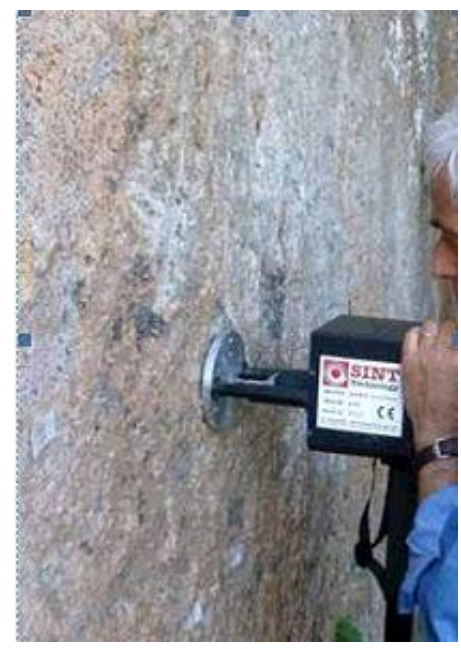

(a)

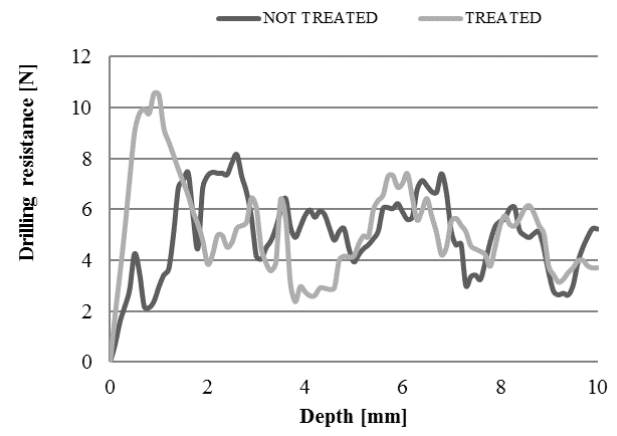

(b)

Figure 12. Archaeological Etruscan area of Sovana. (a) Drilling test and (b) cohesion profiles on the area treated with an acrylic resin

\section{CONCLUSION}

The maintenance and conservation of built heritage and archaeological remains is a complex issue, and to fulfil the objective multidisciplinary competencies are required. The assessment of the state of conservation of the monumental stones is mandatory to establish correct procedures of intervention and define schedule maintenance protocols.

The object in study is considered a unique historic work, and the use of NTD technologies are preferred. The ICVBC furnishes a wide range of competences and such diagnostic techniques to support the field work of monumental heritage restorers, but when it is necessary to verify the performances (penetration, efficiency and durability) of conservation treatments the imaging techniques are out of the scope. In that case we need to have diagnostic tools that are able to furnish reliable data directly correlates with the physical phenomenon: water absorption, in the case of protectives, and the cohesion profiles in the case of consolidants.

\section{AKNOWLEDGMENTS}

We would like to thanks Dr. Francesco Polenta, Head of Administration, and Dr. Anna Galluccio, Scientific Attache' Italian Embassy in Canada, 275 Slater Street, Ottawa, Ontario $\mathrm{K} 1 \mathrm{P} 5 \mathrm{H}$, for the financial support for the organization of the session "Italian Research in Cultural Heritage" in the $26^{\text {th }}$ biennial symposium CIPA2017, Ottawa, Canada.

\section{REFERENCES}

Bandini F., Felici A., Mariotti P.I., Olmi R., Riminesi C., 2008. I dipinti murali della loggia Allori a Palazzo Pitti: una sperimentazione per l'estrazione dei sali e per il monitoraggio con dielettrometria a $\mu$ onde, OPD Restauro, 20, pp. 121-130.

Barbetti, I., Felici, A., Magrini, D., Manganelli Del Fà, R., Riminesi, C., 2013. Ultra close-range photogrammetry to assess the roughness of the wall painting surfaces after cleaning treatments. Int. J. of Cons. Science, 4, pp. 525-534.

Bini, M., Ignesti, A., Olmi, R., Pieri, L., Priori, S., C. Riminesi, C., 2009. US Patent, Patent N. US 7,560,937 B2, Microwave sensor for measuring the moisture content of masonry surfaces comprising a microstrip resonator coupled with an open coaxial probe. Date of Patent: July 14, 2009.

Delgado Rodrigues, J. Costa D., 2004. A new method for data correction in drilling resistance. Tests for the effect of drill bit wear. Int. J. Restor. of Buildings and Monuments, 10, pp. 1-18.

Exadaktylos, G. Tiano, P. and Filareto, C., 2000. Validation of a model of rotary drilling of rocks with the drilling force measurement. Int. J. Restor. of Buildings and Monuments, 3, pp. 307-340.

EN 16581: 2014. Conservation of Cultural Heritage. Surface protection for porous inorganic materials. Lab. test methods for the evaluation of the performance of water repellent product.

Fratini, F., Rescic, S., Tiano, P., 2006. A new portable sys-tem for determining the state of conservation of monumental stones. Mat. and Structures, 39(2), pp. 139-147.

Mecchi A.M., Sansonetti A., Realini M., Poli T., 2008. A Proposal for a common approach in choosing tests for the protocol evaluation of cleaning methods. In: Proc. 11th Int. Cong. On Deter. and Conserv. of Stone. Torun. Poland, pp. 425-433. 
Manganelli Del Fà, R., Riminesi, C., Tiano, P., 2014. Monitoring of surface pattern of artistic and architectural artifacts by means of ultra-close range photogrammetry. In: Proc. of 6th Europ. Symp. on Religious Art, Restoration and Conserv. (ESRARC2014). Ed. Nardini Editore, Firenze, pp. 164-167.

Mascalchi M., Osticioli I., Riminesi C., Cuzman O.A., Salvadori B., Siano S., 2015. Preliminary investigation of combined laser and microwave treatment for stone biodeterioration. Studies in Conservation, Supplement 1: Papers from the Tenth Conference on "Lasers in the Conservation of Artworks", pp. S19-S27.

Olmi R., Bini M., Ignesti A., Priori S., Riminesi C. Felici A., 2006. Diagnostics and monitoring of frescoes using evanescent-field dielectrometry. Measurement Science and Technology, 17(8), pp. 2281-2288.

Olmi R., Riminesi C., 2008. Study of water mass transfer dynamics in frescoes by dielectric spectroscopy. Il Nuovo Cimento, 31C (3), pp. 389-402.

Osticioli, I.; Mascalchi, M.; Pinna, D.; Siano, S., 2013. Potential of chlorophyll fluorescence imaging for assessing bio-viability changes of biodeteriogen growths on stone monuments. Proc. SPIE 8790, Optics for Arts, Architecture, and Archaeology IV, 879003 (May 30, 2013); doi:10.1117/12.2020563

Proietti, N., Capitani, D., Di Tullio V., Olmi R., Priori S., Riminesi C., Sansonetti A, Tasso F., Rosina E., 2015. In: MOdihMA at Sforza Castle in Milan: Innovative Techniques for Moisture Detection in Historical Masonry, Proc. Inter. Conf. “Built Heritage 2013" (Milano, 18-20 XI 2013), Ed. Centro per Conserv. dei Beni Culturali - Politecnico di Milano, pp. 1-6.

Rescic S., Fratini F., Tiano P., 2010. Onsite evaluation of the "mechanical" properties of Maastricht limestone and their relationship with the physical characteristics, in Smith, BJ, Gomez Heras, M., Viles, HA and Cassar, J (eds), Limestone in the Built Environment: Present Day Challenges for the Preservation of the Past, London, Geological Society of London special publications, 331, pp. 203-207.

Riminesi C., Olmi R., 2016. Localized microwave heating for controlling biodeteriogens on Cultural Heritage assets. International Journal of Conservation Science, 7, 281-294.

Singer, B., Hornschild, I. \& Snethlage, R., 2000. Strenght profiles correction functions for abrasive stones. In: Proc. of DRILLMORE, Firenze, Centro Stampa Toscana Nuova: pp. 3542.

Tiano P. and Pardini C., 2004. Valutazione in situ dei trattamenti protettivi per il materiale lapideo. Proposta di una nuova semplice metodologia. ARKOS Scienza e Restauro dell'Architettura, Nardini Editore, 5, Gen/Mar, pp. 30-36.

Vandevoorde D., Pamplona M., Schalm O., Vanhellemont Y., Cnudde V., Verhaeven E. 2009. Contact sponge method: Performance of a promising tool for measuring the initial water absorption, J. of Cultural Heritage, 10(1), pp. 41-47. 